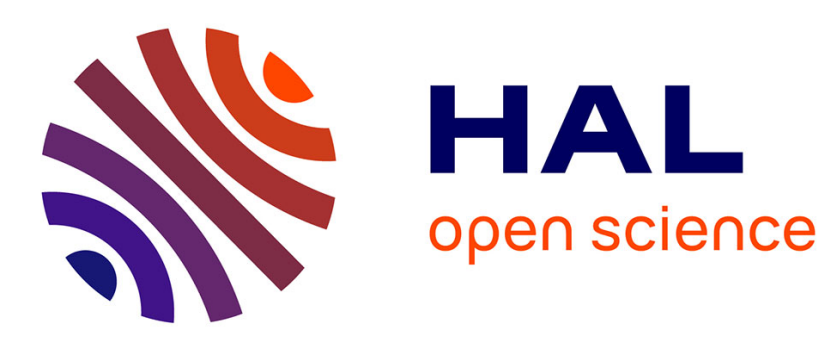

\title{
Plasma sheath material induced dependence due to secondary electron emission
}

\author{
N. Claire, V. Pigeon, N Claire, C. Arnas, K. Terasaka, S. Inagaki
}

\section{To cite this version:}

N. Claire, V. Pigeon, N Claire, C. Arnas, K. Terasaka, et al.. Plasma sheath material induced dependence due to secondary electron emission. Physics of Plasmas, 2020, 27 (4), pp.043505. 10.1063/1.5141348 . hal-02530530

\section{HAL Id: hal-02530530 \\ https://hal-amu.archives-ouvertes.fr/hal-02530530}

Submitted on 3 Apr 2020

HAL is a multi-disciplinary open access archive for the deposit and dissemination of scientific research documents, whether they are published or not. The documents may come from teaching and research institutions in France or abroad, or from public or private research centers.
L'archive ouverte pluridisciplinaire HAL, est destinée au dépôt et à la diffusion de documents scientifiques de niveau recherche, publiés ou non, émanant des établissements d'enseignement et de recherche français ou étrangers, des laboratoires publics ou privés. 


\section{Plasma sheath material induced dependence due to secondary electron emission}

V. Pigeon, ${ }^{\text {a) }}$ N. Claire, and C. Arnas

Aix-Marseille Univ, CNRS, PIIM UMR 7345, 13013 Marseille, France

K. Terasaka

Interdisciplinary Graduate School of Engineering Sciences, Kyushu University, Kasuga, Fukuoka 816-8580,

Japan

S. Inagaki

Research Institute for Applied Mechanics, Kyushu University, 6-1 Kasuga-Koen, Kasuga-city, Fukuoka 816-8580,

Japan

Plasma sheaths in front of six different materials samples $\left(\mathrm{BN}, \mathrm{BNSiO}_{2}, \mathrm{Al}_{2} \mathrm{O}_{3}, \mathrm{SiO}_{2}\right.$, stainless steel and silicon) used in various experiments and devices (Hall thrusters, plasma discharge, microelectronics) are studied using the Laser Induced Fluorescence diagnostic. The specific Secondary Electron Emission (SEE) yield of each material is expected to induce differences in the sheath structure from one sample to another. The experiments are carried out in two different plasma discharges (multipolar device and ECR device), exhibiting distinct electron distribution functions: bi-maxwellian and maxwellian. The agreement between the two experiments is good and allow to classify the materials in a consistent way regarding their SEE yields. The multipolar experiments results are compared to a 1D kinetic sheath model and a $1 \mathrm{D}-1 \mathrm{~V}$ kinetic sheath simulation code. The predictions of the model are discussed and are in good agreement with previous theory. The influence of the low energy impinging electrons on the SEE yield and emissive sheaths is investigated with the code.

\section{INTRODUCTION}

The plasma sheath physics is almost as old as plasma physics itself. It was first described by Langmuir when he was studying discharges in gases in the early 1920 's ${ }^{1}$. Since then, the sheath physics experienced numerous developments, including the pre-sheath physics and Bohm criterion $^{2}$, or the effect of electron emission from the wall on the sheath ${ }^{3}$. The latter is of great importance since all materials naturally emit electrons when facing a plasma. This is the effect of the ions and, mainly, electrons bombardment (in low temperature plasmas at least). This phenomenon is called secondary electron emission $(\mathrm{SEE})^{4}$.

The sheath's modifications triggered by the SEE have several effects. The particles and energy fluxes are affected, which may lead to the heating of the wall material and impact the sputtering yield ${ }^{5}$. The emitted electrons may interact with the plasma and trigger instabilities ${ }^{6}$. The extent of those modifications mainly depends on the material constituting the wall, the plasma temperature and the ions nature. Indeed, the SEE yield is characteristic of each material and is a function of the impinging electron energy ${ }^{7,8}$. Moreover, theoretical and numerical modeling showed that substantial changes in the sheath structure happen when the the emission overpasses a threshold that is dependent on the ion to electron mass ratio ${ }^{3}$. A part (or all) of the sheath electric field may reverse to repel some of the emitted electrons back to the wall. These new sheath structures are respectively called Space-Charge Limited ${ }^{3}$ (SCL) and inverse ${ }^{9}$

a) Electronic mail: valentin.pigeon@univ-amu.fr regimes. These sheath modifications may impact many applications where the plasma-wall interaction plays a major role, like in etching processes, ion implantation experiments, Hall thrusters or in fusion devices.

In the case of Hall thrusters, the plasma faces ceramic walls, mainly made of boron nitride $(\mathrm{BN})$ or borosil $\left(\mathrm{BNSiO}_{2}\right)$. These materials are chosen for their refractory properties, their electrically insulating nature, and their relatively low SEE yields compared to other ceramics ${ }^{10}$ (like alumina). However, the high electron temperature (between 30 and $40 \mathrm{eV}$ ) is able to trigger quite large amounts of SEE which is suspected to be partly responsible for instabilities and anomalous transport that are experimentally observed in thrusters $^{11}$. It was shown that SEE is responsible for the plasma electron temperature decrease, leading to lower performances $^{12}$. Simulations showed that SEE in such discharges can cause oscillations and instabilities of the $\operatorname{sheath}^{13}$.

For these reasons, it is important to precisely know the SEE yields of the ceramics used in the thrusters. These yields are generally measured using electron guns experiments, which are well suited for high energy electrons but not for electrons energies lower than $10 \mathrm{eV}^{14}$. Recently, experiments were performed in low temperature, low density plasma discharges, measuring sheaths in front of Hall thrusters' ceramics ${ }^{16,17}$. These experiments were performed with emissive probes that probably perturb their surroundings (especially plasma sheaths), even if used in low emission regimes. More generally, it is important to have a precise idea of the SEE related to the materials used in plasma experiments. Indeed, the variety of materials used in plasma physics may lead to a variety of situations and plasma sheaths.

Hence, the work presented in this paper provides ion 
velocity distribution functions measurements in front of several materials' sheaths in low temperature, low density plasma discharges taking advantage of the non-intrusive laser-induced fluorescence diagnostic. The Hall thrusters' ceramics are compared to various materials exhibiting different SEE yields, in two different ionization source (multipolar and ECR discharge). The resulting variety of the SEE yields is shown to produce a variety of sheath potential drops in front of the materials samples. The multipolar device's experimental results are compared to a 1D kinetic sheath model and a 1D-1V kinetic sheath simulation code that are introduced in a dedicated section

\section{EXPERIMENTAL SET-UP}

\section{A. Plasma discharge and LIF system}

The first set of experiments is performed in a multipolar device dedicated to LIF experiments ${ }^{18,19}$. The discharge is produced using two Tungsten wires heated to thermionic emission and biased negatively with respect to the grounded vacuum vessel. The filaments emit energetic ionizing electrons that are confined by a magnetic cusps structure created by permanent magnets located at the walls (Figure 1). The plasma at the center of the device is unmagnetized. The used gas is Argon at a $10^{-4}$ mbar working pressure. The discharge current is kept constant at $0.5 \mathrm{~A}$ during all the experiments, and the discharge bias is set to four different values: 50,75 100 and $120 \mathrm{~V}$. The plasma parameters are measured using a Langmuir probe. With these discharge parameters, the electron distribution function is bi-maxwellian: a majority of cold electrons and a small proportion of hot electrons that results from the thermalization of energetic electrons emitted by the filaments ${ }^{20}$. Energetic ionizing electrons might be present, but their amount is too small to be detected here. Cold and hot electrons parameters are obtained from the nonlinear fit of the Langmuir probe's electronic current. The electrons' parameters for the different discharge biases are shown in Figure 2. The cold electron density $n_{e}$ increases from $9 \times 10^{14}$ to $1.25 \times 10^{15} \mathrm{~m}^{-3}$ and the temperature $T_{e}$ remains relatively constant around $1.3 \mathrm{eV}$. The hot electrons temperature $T_{e h}$ rises from 12 to $14.5 \mathrm{eV}$ between 50 and $100 \mathrm{~V}$ and then starts to decreases, while their density $n_{e h}$ monotonically decreases. This is due to the global increase of the plasma density with the discharge bias, resulting in higher collisions' rates that more efficiently thermalize the energetic electrons.

The second set of experiments is performed in the HYPER-II device located in Kyushu University ${ }^{21}$. It is an ECR device: the plasma discharge is provided by a microwave $(2.45 \mathrm{GHz})$ that interacts with the gyromotion of magnetized electrons, giving them energy that is used to ionize Argon gas. A scheme of the device is shown in Figure 3. The plasma is produced in the pro-

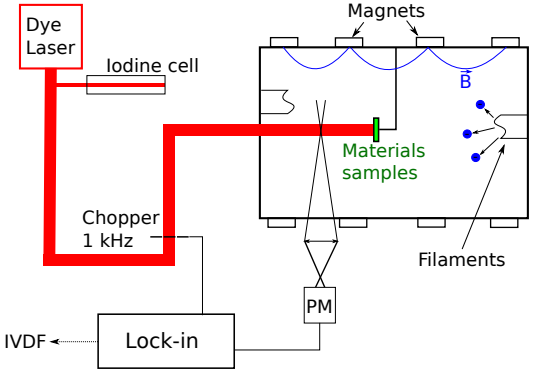

FIG. 1. Scheme of the multipolar device and the LIF set-up. The LIF set-up in HYPER-II is equivalent.

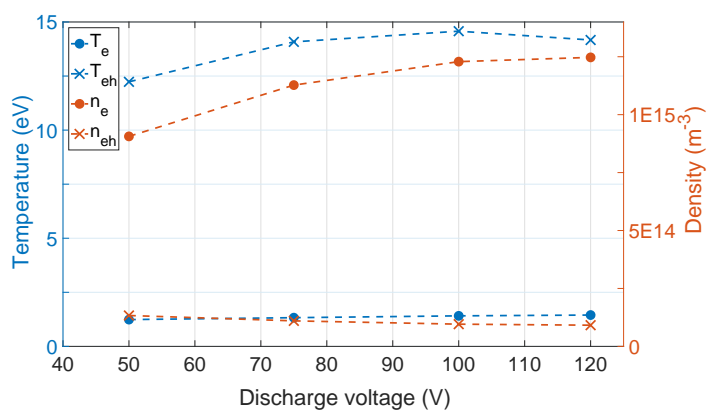

FIG. 2. The hot (index $e h$ ) and cold electrons (index $e$ ) density and temperature for the four different discharge biases used in the experiments.

duction chamber (left part) and diffuses in the large diffusion chamber (right part) where it gets colder and less dense and where the magnetic field decreases $(0.011 \mathrm{~T}$ at the measurement location). Here the electron distribution function is well described by a maxwellian. With the working pressure of $5 \times 10^{-4}$ mbar and a microwave power between 5 and $7 \mathrm{~kW}$, the electron temperature is $\sim 3 \mathrm{eV}$ and the density $\sim 10^{17} \mathrm{~m}^{-3}$. The samples were placed in the diffusion chamber through the bottom left flange (Figure 3) and the laser beam was directly injected through the window on the right side of the device.

The LIF set-ups within the two devices are equivalent and shown in Figure 1. The main difference is the tunable laser, which is a liquid dye laser for the multipolar device and a diode laser for HYPER-II. The liquid dye laser excites the $611.5 \mathrm{~nm}$ metastable transition, while the diode laser excites the $668.1 \mathrm{~nm}$ metastable transition. The liquid dye laser maximum power is 400 $\mathrm{mW}$ (single mode), but it was operated at lower powers to limit the effects and experimental biases due to the 


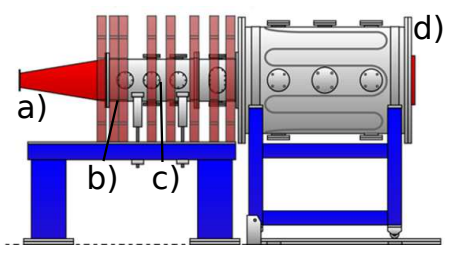

FIG. 3. Scheme of the HYPER-II device. a) RF waveguide. b) Magnetic coils. c) Production chamber. d) Diffusion chamber. The sample holder is fixed on the bottom left flange of the diffusion chamber.

optical pumping saturation ${ }^{22,23}$. The maximum diode laser power is $150 \mathrm{~mW}$. The laser is directly injected inside both devices and propagated perpendicularly to the samples' surface. The LIF signal is detected by a photomultiplier (PM) connected to a lock-in amplifier. The lock-in amplifier reference frequency is given by a chopper (an electro-optical modulator in HYPER-II): $1 \mathrm{kHz}$ in the multipolar device and $100 \mathrm{kHz}$ in Hyper-II. The lock-in signal is recorded using a computer. Slow wavelength ramps are performed with the lasers $(40$ to $60 \mathrm{~s}$, slow compared to the integration time of the lock-in amplifier: $300 \mathrm{~ms}$ ), which directly provides the Ion Velocity Distribution Function (IVDF). The IVDF allows the calculation of the local ion drift velocity due to the presence of the sheath/pre-sheath electric field. In the multipolar device, the PM is mounted on a movable trolley, that allows LIF measurements along the sheath and pre-sheath. The spatial resolution is given by the slit and lens assembly standing in front of the PM and is $0.5 \mathrm{~mm}$. In HYPER-II, the collecting optics and PM are not movable and the measurements were performed at constant distance of the samples (the resolution is $0.5 \mathrm{~mm}$ )

\section{B. Materials' samples}

Six different materials are considered for this study. Three of them are ceramics: boron nitride $(\mathrm{BN})$, borosil $\left(\mathrm{BNSiO}_{2}\right)$ and alumina $\left(\mathrm{Al}_{2} \mathrm{O}_{3}\right)$. The latter is broadly used in plasma experiments while the former two are used in Hall thrusters for their lower SEE yield ${ }^{7}$. These materials' samples consists of $2 \mathrm{~cm}$ diameter disks. Materia samples are at floating potential, glued on an insulated plastic screw. This screw has a diameter 2 times lesser than the samples one. It is fixed on a mechanical support isolated from the vacuum chamber by ceramics. The ceramics samples were cleaned with hydrochloric acid before the experiments to remove the dusts and oxides that may be trapped on the surface. The fourth sample is a stainless-steel disks of same dimensions. The fifth sample consists of a 1 by $2 \mathrm{~cm}$ rectangular piece of quartz $\left(\mathrm{SiO}_{2}\right)$. The last sample is a $1 \mathrm{~cm}$ squared piece of silicon $(\mathrm{Si})$ The samples were chosen for their various SEE yields,

\begin{tabular}{|c|c|c|c|c|c|c|}
\hline \hline Material & $\mathrm{BN}$ & $\mathrm{BNSiO}_{2}$ & $\mathrm{Al}_{2} \mathrm{O}_{3}$ & $\mathrm{SiO}_{2}$ & Steel & $\mathrm{Si}$ \\
\hline Nature & Ins. & Ins. & Ins. & Ins. & Cond. & Semi-cond. \\
\hline$E_{1}(\mathrm{eV})$ & 45 & 40 & 22 & 22 & 40 & - \\
\hline \hline
\end{tabular}

TABLE I. Summary of the different materials electrical properties (insulator, conductor, semi-conductor) and $1^{\text {st }}$ crossover energy $E_{1}$. There is no first crossover energy for the silicon as its yield never get higher than $0.9^{8}$

$\gamma$, and electrical properties: four insulators, one conductor and one semi-conductor. The SEE yields awailable in the literature: $\mathrm{BNSiO}_{2}, \mathrm{BN}$, and $\mathrm{Al}_{2} \mathrm{O}_{3}$ yields have been experimentally measured in vacuum conditions ${ }^{7}$; the steel's yield was experimentally obtained in plasma conditions ${ }^{25}$; the $\mathrm{SiO}_{2}$ and $\mathrm{Si}$ yields are theoretical ${ }^{8}$. The variations between the SEE yields can be shown by the first crossover energy, i.e. the impinging electron energy that corresponds to $\gamma=1$. This is summarized in Table I. The variety of SEE yields is expected to produce a variety of plasma sheaths in front of the samples since higher SEE yields lead to lower sheath potential drops.

\section{THEORETICAL AND NUMERICAL MODELING OF THE SHEATH IN THE PRESENCE OF SEE}

To compare the sheath potential drops deduced from the multipolar device experiments, a 1D kinetic steadystate sheath model and a $1 \mathrm{D}-1 \mathrm{~V}$ kinetic simulation code were developed. They both model the sheath physics in the presence of secondary electron emission.

\section{A. 1D kinetic sheath model}

The 1D kinetic sheath model is inspired by the one presented in Ref 15 and 16 . It models the steady-state sheath in front of a wall immersed in a multipolar plasma discharge. The pre-sheath is not considered and the potential at the sheath entrance is set to zero. To model the depletion of particles because of the presence of the wall, the distribution functions are truncated. The model is made of four equations: the neutrality at the sheath edge, the flux balance at the wall (the floating wall hypothesis), the SEE equation and the generalized Bohm criterion. Several changes with respect to the initial model were performed. Since the presence of the ionizing electrons in the discharge was not detectable in our case, the electron distribution function is implemented as a biMaxwellian in the model. The hypothesis of monoenergetic ions was discarded and a truncated Maxwellian distribution function was implemented instead ${ }^{26}$. The most important change with respect to the previous model ${ }^{16}$ was the implementation of an energy dependent SEE yield. The experimental yields available in the literature $\left(\mathrm{Al}_{2} \mathrm{O}_{3}, \mathrm{BN} \text { and } \mathrm{BNSiO}_{2}\right)^{7}$ are fitted using a power function $\gamma(E)=\delta E^{\sigma}$ (Figure 7 ). This yield is integrated over 


\begin{tabular}{|c|c|c|}
\hline \hline & $\delta$ & $\sigma$ \\
\hline $\mathrm{BN}$ & 0.0819 & 0.6600 \\
\hline $\mathrm{BNSiO}_{2}$ & 0.1394 & 0.5432 \\
\hline $\mathrm{Al}_{2} \mathrm{O}_{3}$ & 0.2179 & 0.5099 \\
\hline \hline
\end{tabular}

TABLE II. Parameters used for the power fitting of the SEE yields for the three different ceramics.

the electron distribution function at the wall $\left(\Phi=\Phi_{w}\right)$ to get the effective SEE yield:

$$
\Gamma_{s}\left(\Phi_{w}\right) \gamma_{e f f}=\left(\int \gamma\left(\frac{1}{2} m_{e} v_{e}^{2}\right) v_{e} f_{s}\left(v_{e}\right) \mathrm{d} v_{e}\right)_{\Phi=\Phi_{w}}
$$

The emitted electron flux $\Gamma_{e e}$ is the linear combination of the different electron fluxes $\Gamma_{s}$ and their corresponding effective SEE yield $\gamma_{e f f}$. It must be noted that the SEE yield is not a power function, especially at energies higher than a few hundreds of $\mathrm{eV}$. It reaches a peak and then decreases with the increasing impinging electron energy. However, considering the energies and temperatures of the electrons in the multipolar plasma $(\leq 30 \mathrm{eV})$, the electron distribution function rapidly reaches negligible values before the SEE yield moves away from a power function and the integrand in Equation 1 vanishes. The parameters used for the power fitting for alumina, boron nitride and borosil are given in Table II.

With these new implementations, the model equations may be written as follows. The quasi-neutrality at the sheath entrance is $N_{i 0}=(\alpha+\eta) N_{e 0}+N_{e e 0}$ where $N_{i 0}$ is the ion density at the sheath entrance, $\alpha$ and $\eta$ represent the relative proportions in the bulk plasma of hot and cold electrons respectively, $N_{e 0}$ is the electron density at the sheath entrance and $N_{e e 0}$ the emitted one. This equation becomes:

$$
0=1-(\alpha+\eta) N_{e 0}-N_{e e 0}
$$

with densities normalized by $N_{i 0}$. The particle fluxes balance, obtained by calculating $\int v_{s} f_{s}\left(v_{s}\right) \mathrm{d} v_{s}$ at the wall for each distribution function, reads:

$$
\begin{aligned}
0= & \frac{1}{\sqrt{\mu \theta_{i}}} \frac{\exp \left(-\theta_{i} \varepsilon\right)}{\operatorname{erfc} \sqrt{\theta_{i} \varepsilon}}-\eta \frac{\exp \left(-\Phi_{w}\right)}{2-\operatorname{erfc} \sqrt{\Phi_{w}}} N_{e 0} \\
& +\frac{1}{\sqrt{\theta_{e e}}} \frac{\exp \left(-\theta_{e e} \Phi_{w}\right)}{\operatorname{erfc} \sqrt{\theta_{e e} \Phi_{w}}} N_{e e 0} \\
& -\frac{\alpha}{\sqrt{\theta_{h}}} \frac{\exp \left(-\theta_{h} \Phi_{w}\right)}{2-\operatorname{erfc} \sqrt{\theta_{h} \Phi_{w}}} N_{e 0}
\end{aligned}
$$

With $\theta_{s}=T_{e} / T_{s}$ the ratio between the cold electron temperature and the other temperatures (ions, emitted electrons and hot electrons); $\varepsilon$ is the ion energy at the sheath entrance; $\Phi_{w}=-\frac{e \phi_{w}}{k_{B} T_{e}}$ the normalized wall potential; $\mu$ the ion to electron mass ratio.
The next model equation indicates that the secondary electron flux at the wall is equal to the sum of the cold and hot electron fluxes at the wall with their corresponding effective electron emission yield derived from eq. 1. The SEE triggered by the ions is not taken into account. Hence, the secondary electron emission equation reads:

$$
\begin{aligned}
0= & \frac{1}{\sqrt{\theta_{e e}}} \frac{\exp \left(-\theta_{e e} \Phi_{w}\right)}{\operatorname{erfc} \sqrt{\theta_{e e} \Phi_{w}}} N_{e e 0} \\
& -\eta \delta \Gamma(1+\sigma) T_{e}^{\sigma} \frac{\exp \left(-\Phi_{w}\right)}{2-\operatorname{erfc} \sqrt{\Phi_{w}}} N_{e 0} \\
& -\frac{\alpha}{\sqrt{\theta_{h}}} \delta \Gamma(1+\sigma) T_{e h}^{\sigma} \frac{\exp \left(-\theta_{h} \Phi_{w}\right)}{2-\operatorname{erfc} \sqrt{\theta_{h} \Phi_{w}}} N_{e 0}
\end{aligned}
$$

Here $\mathbb{\Gamma}$ is the Gamma function. The generalized Bohm criterion is given by $\left.\frac{\mathrm{d} N_{i}}{\mathrm{~d} \Phi}\right|_{\Phi=0}-\left.\sum_{e} \frac{\mathrm{d} N_{\mathrm{s}}}{\mathrm{d} \Phi}\right|_{\Phi=0}=0$ where the subscript 's' denotes ions, electrons or emitted electrons. The marginal solution reads ${ }^{27}$ :

$$
\begin{aligned}
0= & \theta_{i}\left(1-\frac{1}{\sqrt{\pi}} \frac{\exp \left(-\theta_{i} \varepsilon\right)}{\sqrt{\theta_{i} \varepsilon} \operatorname{erfc} \sqrt{\theta_{i} \varepsilon}}\right) \\
& +\eta\left(1-\frac{1}{\sqrt{\pi}} \frac{\exp \left(-\Phi_{w}\right)}{\sqrt{\Phi_{w}} \operatorname{erfc} \sqrt{\Phi_{w}}}\right) N_{e 0} \\
& +\theta_{e e}\left(1-\frac{1}{\sqrt{\pi}} \frac{\exp \left(-\theta_{e e} \Phi_{w}\right)}{\sqrt{\theta_{e e} \Phi_{w}} \operatorname{erfc} \sqrt{\theta_{e e} \Phi_{w}}}\right) N_{e e 0} \\
& +\alpha \theta_{h}\left(1-\frac{1}{\sqrt{\pi}} \frac{\exp \left(-\theta_{h} \Phi_{w}\right)}{\sqrt{\theta_{h} \Phi_{w}} \operatorname{erfc} \sqrt{\theta_{h} \Phi_{w}}}\right) N_{e 0}
\end{aligned}
$$

Numerically solving these four equations for a given set of parameters $\alpha, \eta$ and $\theta_{s}$ leads the obtainment of the unknown parameters $\Phi_{w}$, the wall potential, $E_{w}$ the wall electric potential and the total effective SEE yield $\gamma_{\text {tot }}$ given by :

$$
\gamma_{t o t}=\frac{\sum_{s} \gamma_{s} \Gamma_{s}\left(\Phi_{w}\right)}{\sum_{s} \Gamma_{s}\left(\Phi_{w}\right)}
$$

Figures 4, 5 and 6 represents the solutions $E_{w}, \Phi_{w}$ and $\gamma_{t o t}$ for various parameters. It is therefore possible to verify Hobbs and Wesson's prediction ${ }^{3}$ : the wall electric field vanishes when the SEE yield reaches the critical value given by $\gamma_{\text {crit }}=1-8.3 \sqrt{\frac{m_{e}}{m_{i}}}$. This is known as the SCL (Space-Charge Limited) transition. If the emission is increased beyond this threshold, the wall electric field reverses and a virtual cathode appears and reflects some of the emitted electrons back to the wall. The model is valid for SEE yield lower or equal to the critical threshold. This verification has been performed for a $\mathrm{BN}$ wall in a bi-maxwellian plasma. The cold electron temperature was kept constant $T_{e}=2 \mathrm{eV}$ while the hot electrons temperature and relative density were varied on ranges that are consistent with the multipolar plasma discharges parameters. The mass ratio was the Argon one, leading to a critical SEE yield $\gamma_{\text {crit }}=0.98$. The wall electric field 


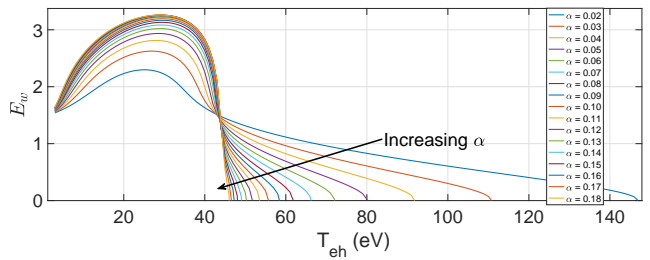

FIG. 4. 1D kinetic sheath model normalized wall electric field $E_{w}$ with respect to the hot electrons temperature $T_{e h} . \alpha$ increases from 0.02 to 0.18 . The wall material is $\mathrm{BN}$.

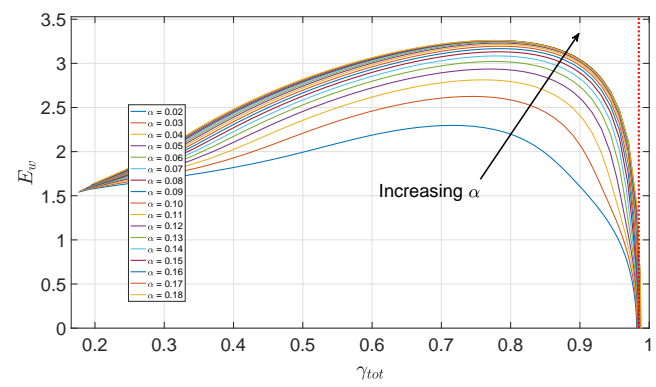

FIG. 5. 1D kinetic sheath model wall electric field with respect to the total effective SEE yield, for different value of $\alpha$. The red dotted line represents the critical emission value $\gamma_{\text {crit }}$

with respect to the hot electrons temperature $T_{e h}=\frac{T_{e}}{\theta_{h}}$ and the self-consistently calculated SEE total effective yield $\gamma_{\text {tot }}$ is shown in Figures 4 and 5 .

It appears that $\gamma_{\text {tot }}$, self-consistently calculated from the BN experimental SEE yield, reaches the critical value $\gamma_{\text {crit }}=0.98$ when the wall electric field vanishes. This is consistent with Hobbs and Wesson theory that was derived for a simple maxwellian electron distribution function. The wall electric field variations can be divided into two different parts. The first part corresponds to the case where the wall electric field increases with the hot electron energy: the sheath physics is dominated by the hot electrons, the wall electric field grows to confine the hot electrons and maintain the particle flux balance. In the mean time, the SEE yield increases: there is a competition between the hot electrons and the emitted electrons. In the second region, the wall electric field decreases with the hot electron temperature and eventually reaches zero. In this case the sheath physics is dominated by the emitted electrons: the emission becomes large enough so the sheath starts to collapse. The transition between the two parts occurs for temperatures between 25 and $30 \mathrm{eV}$, depending on the hot electrons relative density $\alpha$, which corresponds to $\gamma_{\text {tot }}$ values between

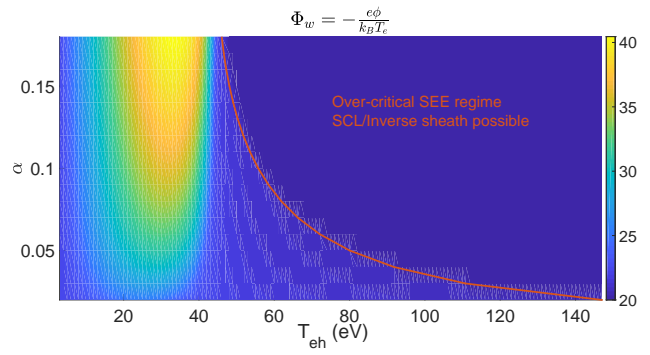

FIG. 6. 1D kinetic sheath model sheath potential drop in front of the BN sample with respect to $T_{e h}$ and $\alpha$. The input parameters are the same as in Figure 4 and 5 . The red line corresponds to the SCL transition region $\left(\gamma_{\text {tot }}=\gamma_{\text {crit }}\right)$. The model cannot compute the potential value above this line, which was set to 0 .

0.71 and 0.78 . The effective SEE yield value corresponding to the vanishing electric field slightly increase with $\alpha$ (from 0.981 to 0.988 ). The increase of $\alpha$ reduces the temperature needed to reach the critical electron emission: higher emission yields are reached for lower temperatures. Here the wall electric field, as well as the wall potential is never multi-valued as it was observed in Langendorf's work $^{16}$. It seems that this feature is linked to the presence of isotropic mono-energetic electrons ${ }^{28}$ that are not present in this multipolar device modeling.

Regarding the hot electrons temperature and relative density ranges available in the multipolar device (from 10 to $20 \mathrm{eV}$ and from 0.01 to 0.15 , respectively) it is highly improbable that the sheath in front of the ceramics collapses and reaches the SCL transition. The sheath potential drop with respect to the $T_{e h}$ and $\alpha=\frac{N_{e h}}{N_{e}}$ is shown in Figure 6. The SCL transition region is shown in red. $T_{e h}$ and $\alpha$ available in the multipolar device are far from this transition region, so the sheath should remain classical with relatively high sheath potential drops.

\section{B. 1D-1V kinetic simulations}

Along with the kinetic 1D model, a 1D-1V sheath simulation code inspired from the work of Campanell ${ }^{29}$ has been developped. The global structure of the code is the same: it simulates a plasma bounded by two walls, that may emit electrons, by solving the Boltzmann-Poisson equations. The collision and source operators have not been modified and their input parameters were kept the same. The source operator injects at each time step a number of particles equal to the amount reaching the walls. The electron collisions operator relaxes the distribution function toward the initial one (BGK operator); the ion collision operator models charge exchange collisions and replaces fast ions by slow ones. The length 
of the plasma is $10 \mathrm{~cm}$ (the walls located at $x=0$ and $x=10 \mathrm{~cm}$ ), the charge-exchange mean free path is set to $5 \mathrm{~cm}$, the numerical grid dimension is $4096 \times 1024$ ( $x$ and $v$ coordinate respectively). The working gas was Argon. The maximum values for the velocities are $\left(v_{e} / v_{\text {the }}\right)_{\max }=11$ and $\left(v_{i} / c_{s}\right)_{\max }=8.5$. The time step was adjusted regularly during the simulations while being consistent with the CFL condition. In Campanell's simulations, the electron emission is set to arbitrary levels. Here the SEE is calculated self-consistently using experimental data similarly to the $1 \mathrm{D}$ kinetic model. The maxwellian electron distribution function is replaced by a bi-maxwellian one to model the multipolar plasma. Unlike the model, the simulations allow the study of the pre-sheath and the temporal dynamic of the sheath.

The code may also be used to study the influence of the low energy impinging electrons on the yields. Discussions about this feature started fifteen years ago with the article of $\mathrm{Cimino}^{30}$ that showed that the SEE yield of particles accelerators copper walls may reach 1 when the energy of the impinging electrons goes to 0 . Further works aimed to show that it should not be the case ${ }^{31}$, while recent works showed it may actually happen for dirty metallic surfaces ${ }^{32}$. New theoretical models mixing surface and plasma physics showed that this feature may also exist for insulators ${ }^{33}$ like $\mathrm{Al}_{2} \mathrm{O}_{3}{ }^{34}$. This SEE at low impinging energy may have an influence on the sheath potential drop and reduce it by a factor $10 \%^{34}$. It may also lower the electron temperature/energy threshold needed to reach the SCL transition for the strongest emitters.

To investigate this effect, a simple fitting algorithm was used to extend the experimental SEE yields to very low energies. This fitting does not take into account all the physics that has been derived in the recent theoretical models. However, it does allow one to mimic the SEE yield behavior that is expected from this theory. An exponential contribution was added to the power fitting, leading to SEE yields close $0.5-0.6$ when the impinging electron energy goes to zero. The two fitting algorithm are shown in Figure 7. This behavior can be guessed in Tondu's experimental data ${ }^{7}$ despite measuring the SEE yields for energy lower than $10 \mathrm{eV}$ is hardly achievable ${ }^{14}$.

\section{MATERIALS' COMPARISON}

\section{A. Multipolar experiments}

The IVDFs were measured in the sheath and presheath standing in front of each materials listed in Table I for the four discharge biases $(50,75,100,120 \mathrm{~V})$. The ion drift velocity was calculated using the formula $V_{i}=\frac{1}{n_{i}} \int v_{i} f_{i}\left(v_{i}\right) \mathrm{d} v_{i}$, with $n_{i}=\int f_{i}\left(v_{i}\right) \mathrm{d} v_{i}$. The results presented in Figure 8 to 11 show the drift velocity with respect to the wall distance. The corresponding potential is not calculated as no hypothesis on the collisionality has been made. However the differences in the velocities,

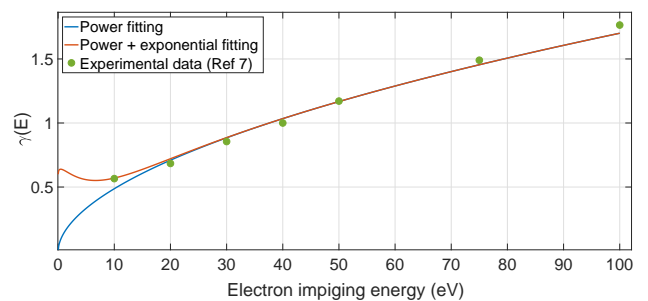

FIG. 7. Fittings of the $\mathrm{BNSiO}_{2} \mathrm{SEE}$ yield experimental data of Ref 7 .

especially the drift velocity at the wall allows one to characterize differences between the materials' sheaths.

First, it appears that the different materials exhibit a variety of wall drift velocities, i.e. a variety of sheath potential drops. Also, the sheath thickness varies from one material to another. The sheath entrance corresponds to the location where the drift velocity reaches the Bohm velocity (black dotted horizontal line). Hence, these results confirm that the sheath is material dependent. The sheath size and potential drop does not only depend on plasma parameters like the ion to electron mass ratio. Regarding the wall velocities, it is possible to classify the materials in three different groups: those with the largest velocities (red lines), those with lower velocity (blue lines) and the last one with the lowest wall velocity (green).

The first group is made up of $\mathrm{BN}, \mathrm{BNSiO}_{2}$ and stainless steel. These materials exhibit large wall velocities that are close to each other. This is consistent with the experimental data ${ }^{7,25}$. These materials have comparable SEE yields and first crossover energies (Table I). It is therefore expected that they should have similar sheath potential drops, which is what is observed here.

The second group is made of $\mathrm{Al}_{2} \mathrm{O}_{3}$ and $\mathrm{SiO}_{2}$. The sheath potential drops in front of these materials are similar, which is consistent with their close first crossover energy and SEE yields. Their higher SEE yields at constant energy than the first group lead to a lower sheath potential drop. Indeed, a higher emission reduces the sheath potential drop to maintain particle flux balances by allowing more plasma electrons to fall onto the surface. This result confirms the choice of using BN-like ceramics instead of alumina in Hall thrusters since it is necessary to limit the SEE in those devices.

The last group is made only of silicon. The fact that this material exhibit very low sheath potential drops is quite surprising. Indeed, it has the lowest emission of the materials considered in this study (it does not have a first crossover energy since its SEE yields always remains lower than 1$)^{8}$. Two hypotheses can be made to explain this peculiar behavior. The first one is the positive surface charging of the silicon surface due the 


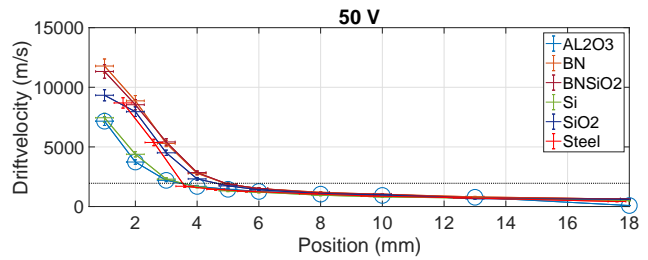

FIG. 8. Drift velocities measured in front of the samples for the $50 \mathrm{~V}$ discharge. The black line corresponds to the Bohm velocity $(1900 \mathrm{~m} / \mathrm{s})$. Measurements points are indicated only once by a circle and are connected by lines.

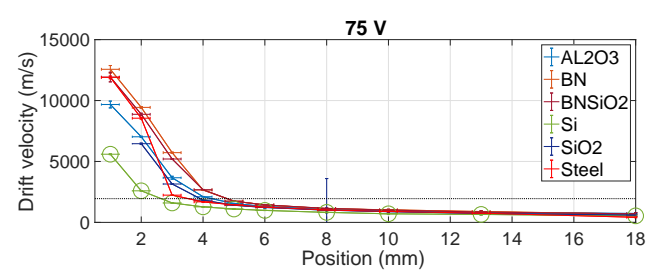

FIG. 9. Drift velocities measured in front of the samples for the $75 \mathrm{~V}$ discharge. The black line corresponds to the Bohm velocity $(1900 \mathrm{~m} / \mathrm{s})$. Measurements points are indicated only once by a circle and are connected by lines.

ions. This was observed in ion implantation experiments in RF discharges ${ }^{35}$. The ion implantation energy was decreased during the duration of the pulse because the ions got trapped inside the silicone surface, reducing the surface negative charge and hence the positive sheath space-charge and sheath potential drop. This issue was addressed by reducing the pulse duration, allowing the surface to neutralize during the pulse-off. Here, the multipolar provides a steady-state plasma discharge. There would therefore be no way for the ions to escape the silicon surface. The second hypothesis that could explain the low sheath potential drops would be the presence of oxides at the silicon surface, that could greatly modify the SEE yield and therefore the sheath structure. The yields of such oxides cannot be easily found in the literature, limiting the verification of this hypothesis as well.

The global increase of velocities at the wall with the discharge bias is consistent with the model's predictions (Section III.A). The temperature of the hot electrons increases with the discharge voltage. In this range of temperature, the model predicts that the sheath physics is dominated by the hot electrons so the sheath potential drops increases with the temperature, which is what is observed.

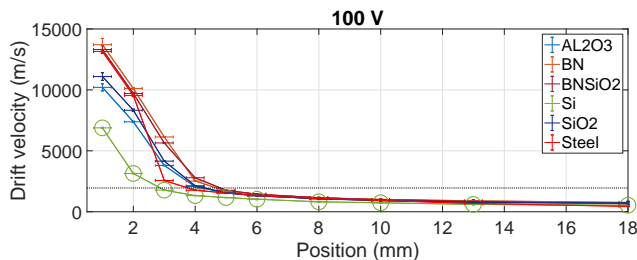

FIG. 10. Drift velocities measured in front of the samples for the $100 \mathrm{~V}$ discharge. The black line corresponds to the Bohm velocity $(1900 \mathrm{~m} / \mathrm{s})$. Measurements points are indicated only once by a circle and are connected by lines.

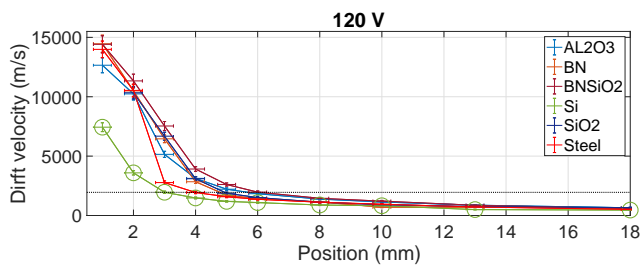

FIG. 11. Drift velocities measured in front of the samples for the $120 \mathrm{~V}$ discharge. The black line corresponds to the Bohm velocity $(1900 \mathrm{~m} / \mathrm{s})$. Measurements points are indicated only once by a circle and are connected by lines.

\section{B. HYPER-II experiments}

LIF measurements in front of the materials were performed in HYPER-II as well. This set of experiments allowed for the measurement of IVDFs in this device for the first time. As described in Section II, the LIF detection system is not movable in this device. It was therefore chosen to perform the measurements at a constant distance for all the samples. This distance close to $1 \mathrm{~mm}$ corresponds approximately to the ion acoustic speed for BN. The BN sample was first placed inside the diffusion chamber. The detection system (that can be slightly moved around its position) was placed such that the measured ion drift velocity approximately corresponds to the Bohm velocity (which was about $2800 \mathrm{~m} / \mathrm{s}$ in the considered discharge). The IVDFs were measured for three different RF input powers $(5,6$ and $7 \mathrm{~kW})$, and the samples replaced sequentially. The results are shown in Figure 12.

The RF input power increases the plasma density, thus reducing the sheath thickness. As a result, the sheath entrance is displaced toward the sample, and the measured drift velocity at constant distance decreases with the RF power.

The hierarchy that was found in the multipolar device also appears in HYPER-II. The three groups can be 


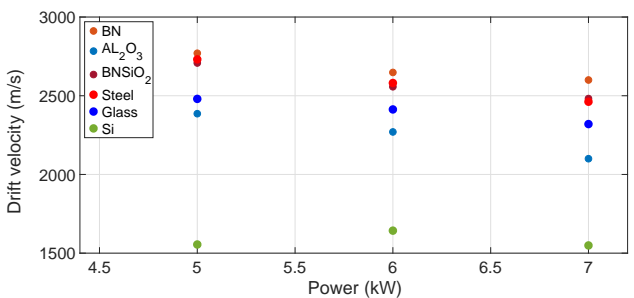

FIG. 12. Ion drift velocity measured at constant distance of all the samples in HYPER-II, for three different RF input powers. The error bars are not shown for clarity and are about $200 \mathrm{~m} / \mathrm{s}$.

identified: $\mathrm{BN}, \mathrm{BNSiO}_{2}$ and steel (red); $\mathrm{Al}_{2} \mathrm{O}_{3}$ (blue) and $\mathrm{SiO}_{2}$ (green); silicon. It appears that the sheath material dependency prevails here as well, even if the electron distribution function is purely maxwellian and the temperature quite low. Differences in the SEE yields lead to observable differences in the sheath potential drop. This dependency may effect all the plasma discharges and should always be considered, especially in the case of experiments dealing with plasma-wall interactions.

\section{Theoretical and numerical results compared to the multipolar devices measurements}

The LIF measurements in the multipolar device were eventually compared to those obtained with the $1 \mathrm{D}$ model and the 1D-1V simulations. Only three of the materials are used for the comparisons: $\mathrm{BN}, \mathrm{BNSiO}_{2}$ and $\mathrm{Al}_{2} \mathrm{O}_{3}$, especially because experimental SEE yields are available for them ${ }^{7}$. The multipolar plasma parameters were put in the model and simulations. The comparison concerns the sheath potential drop. For the experimental data, it was calculated as $\phi_{s}=\frac{1}{2 e} m_{i}\left(v_{w}^{2}-c_{s}^{2}\right)=$, with $v_{w}$ the wall velocity. The wall velocity was not measurable and the smallest wall distance's drift velocity was used instead. This leads to slightly undervalued experimental sheath potential drops. In the model the sheath potential drop is merely the wall potential, since the plasma potential at the sheath entrance is set at 0 . In the simulations the sheath potential drop was calculated between the wall and the location where the ions reach $c_{s}$. The results are shown in Figure 13.

The agreement between the experiments, the model and the simulations is correct for the $\mathrm{BNSiO}_{2}$ and $\mathrm{BN}$. The model gives slightly larger values for the sheath potential drop. This is due to the fact that there are two walls in the simulations. The emitted electrons are accelerated and form a beam that propagate inside the plasma. This beam is not fully thermalized before reaching the opposite wall and colliding with it. This small

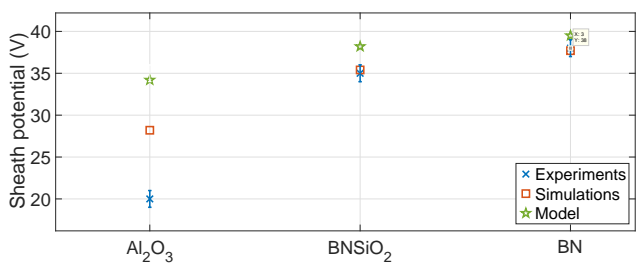

FIG. 13. Comparison of the sheath potential drops obtained with the experiments, the $1 \mathrm{D}$ model and the $1 \mathrm{D}-1 \mathrm{~V}$ simulations.

beam slightly increases the SEE while the plasma electrons temperature remains constant. As a result, the sheath potential drop is decreased. The more emissive the material, the larger the decreased.

On the other hand, the agreement is poor for the alumina sample. The difference between the model and the simulation can be explained by the same mechanism explained previously. Here, however, the experimentally measured sheath potential is lower than the simulations (and hence the model's agreement). This may have two causes. The first would be that the SEE yield has been underestimated. The second would be the influence of the low energy impinging electrons on the SEE. As stated in paragraph III B, it was shown that alumina's SEE yield may not go to zero with the impinging electron energy.

To investigate this, the new yield fitting was used in the simulations. It was first tested on the $\mathrm{BN}$ and $\mathrm{BNSiO}_{2}$. Using this new yield decreases the sheath potential drop by a factor $10 \%$ and increases of the effective SEE yield by $20 \%$. This is consistent with the values given by the recent theoretical models ${ }^{34}$. Since the plasma electrons falling onto the wall are slowed down by the sheath electric field, their mean energy decreased toward zero. Therefore, a higher SEE yield at low impinging electron energy enhance the modifications of the sheath potential drops.

The same simulation was performed for the $\mathrm{Al}_{2} \mathrm{O}_{3}$ sample. Comparisons with the model and experiments are not possible with the present simulations. Indeed, it appears that the increased SEE triggers instabilities. The emitted electron beam interacts with the bi-maxwellian electrons, leading to bump-on-tail instabilities that create periodic oscillations of the electron density and electric potential in the bulk plasma. Moreover, the interaction of the emitted electron beam with the facing wall, that was lowering the sheath potential in the previous simulations, makes the whole sheath potential unstable. No stable sheath is obtained in these simulations, and new simulations with different plasma geometries (only one wall) would be required to make comparisons with the other results. This will be assessed in a future dedicated work. 


\section{v. CONCLUSION}

The sheath in front of different wall materials (BN $\mathrm{BNSiO}_{2}, \mathrm{Al}_{2} \mathrm{O}_{3}, \mathrm{SiO}_{2}$, stainless steel and silicon) was performed with experimental, theoretical and numerical techniques The LIF experiments were performed in two different plasma discharges: a multipolar device and a ECR plasma. The theoretical study was performed with a steady-state 1D sheath model, and numerical analysis part was done using a $1 \mathrm{D}-1 \mathrm{~V}$ sheath simulation simulation code.

The experiments showed several features. The first and foremost is the confirmation that the sheath is material dependent. The sheath potential drop and thickness differs from one material to another. Here, these differences have been identified to be mainly due to the SEE. These differences should be taken into account in experiments and simulations, since the sheath potential drop may vary by a factor 2 from one material to another and may have an impact on the physics inside the bulk plasma.

A hierarchy between the wall materials has been established from the consistent results obtained in the two plasma discharges. A first group of materials, made of $\mathrm{BN}, \mathrm{BNSiO}_{2}$ and stainless steel exhibit similar sheath potential drops that are the largest of all the samples. This is consistent with the similar SEE yields of these materials. The second group is made of $\mathrm{Al}_{2} \mathrm{O}_{3}$ and $\mathrm{SiO}_{2}$. Their sheath potential drops are lower than the first group's which is consistent with their higher SEE yields. This confirms the choice of $\mathrm{BN}$ and $\mathrm{BNSiO}_{2}$ instead of alumina as Hall thrusters' wall materials, since it is necessary to limit the SEE is these devices. The last group consists solely of silicon that has the lowest sheath potential drop. This cannot be explained by the SEE and could be explained by the positive ion charging of the surface or the presence of oxides at the sample surface. This last result needs further investigations in order to be confirmed.

The 1D model provides a description of the sheath in the presence of SEE in a bi-maxwellian plasma that is consistent with Hobbs and Wesson's theory. The total effective SEE yields, computed from the fitting of experimental data, reaches the critical value $\gamma_{\text {crit }}=0.98$ when the wall electric field vanishes. The model predictions of the sheath potential drop evolution with the plasma electrons parameters are consistent with the experimental results. A 1D-1V simulation code has been developed. It is able to investigate the influence of the low energy impinging electrons on the SEE, which has been recently shown to be considerable and to have an influence on the sheath structure.

The comparisons between the experiments, the model and the simulations show good agreements with $\mathrm{BN}$ and $\mathrm{BNSiO}_{2}$. The agreement is quite poor with $\mathrm{Al}_{2} \mathrm{O}_{3}$. The possible influence of the low energy impinging electrons on the SEE has been investigated in address these differences. It appears that the enhanced electron emission triggers instabilities that prevent comparison between the simulations results and the experiments results. These instabilities need a dedicated study in further works.

The study presented in this paper can be extended to any materials relevant in plasma experiments. Measurements in front of copper samples could be interesting to compare with the results presented in Cimino's paper. It is also possible to study fusion relevant materials for instance, since it is mandatory to precisely know the emission properties of the wall materials in these devices. Further measurements can also be performed for the various silicon samples (undoped, doped, clean, dirty) to explain the results obtained in the present study.

\section{ACKNOWLEDGMENT}

The authors would like to thank Ms E. Abe for her technical support; Dr. Muraglia, Dr. Poye and Pr. Agullo for their precious help concerning the development of the simulation tools. This work is partly supported by the JSPS Postdoctoral Fellowships (JSPS Summer Program: SP18216) and by the collaboration programs of NIFS (NIFS18KNWP007)

\section{REFERENCES}

${ }^{1}$ I. Langmuir, Science 58(1502), 290 (1923).

${ }^{2}$ A. Guthrie and R. K. Wakerling, The Characteristics of Electrical Discharges in Magnetic Fields (McGraw-Hill Book Company,

${ }^{3}$ G. D. Hobbs and J. A. Wesson, Plasma Phys. 9(1), 85 (1967).

${ }^{4}$ M. A. Furman and M. T. F. Pivi, Phys. Rev. Special Topics Accelerator and Beams 5(12), 124404 (2002).

${ }^{5}$ J. Ou and X. Zhao, Contrib. Plasma Phys. 57(2), 50 (2016)

${ }^{6}$ M. C. Griskey and R. L. Stenzel, Phys. Rev. Lett. 82(3), 556 (1999)

${ }^{7}$ T. Tondu, M. Belhaj and V. Inguimbert, J. Appl. Phys. 10(9), 093301 (2011).

${ }^{8}$ J. Seon and E. Lee, Plasma Sci. Tech. 15(11), 1093.

${ }^{9}$ M. D. Campanell, Phys. Rev. E 88(3) (2013).

${ }^{10}$ D. M Goebel and I. Katz, Fundamentals of Electric Propulsion: Ion and Hall Thrusters (John Wiley \& Sond Inc., 2008).

${ }^{11}$ J-P. Boeuf, J. Appl. Phys. 121(1), 011101 (2017).

${ }^{12}$ Y. Raitses, A. Smirnov, D. Staack and N. J. Fisch, Phys. Plasmas 13(1), 014502 (2006)

${ }^{13}$ M. D. Campanell, A. V. Khabrov and I. D. Kaganovich, Phys. Rev. Let. 108(23), 235001 (2012).

${ }^{14}$ J. Cazaux, J. Appl. Phys. 111(6), 064903 (2012)

${ }^{15}$ J.P. Sheehan, N. Hershkowitz, I. D. Kaganovich, H. Wang, Y. Raitses, E. V. Barnat, B. R. Weatherford, and D. Sydorenko,f and M. Walker, Phys. Rev. Lett. 111, 075002 (2013).

${ }^{16}$ S. Langendorf and M. Walker, Phys. Plasmas 2(3), 033515 (2015).

${ }^{17}$ I. Schweigert, T. S. Burton, G. B. Thompson, S. Langendorf, M. L. R. Walker and M. Keidar, Plasma Sour. Sci. Tech. 27(4), 045004 (2018).

${ }^{18}$ M. Carrere, L. Chérigier, C. Arnas-Capeau, G. Bachet and F. Doveil, Rev. Sci. Instrum. 67(12), 4124-4129 (1996).

${ }^{19}$ N. Claire, G. Bachet, U. Stroth and F. Doveil, Phys. Plasmas 13(6), 062103 (2006)

${ }^{20}$ E. Stamate, K. Inagaki, K. Ohe and G. Popa, 32(6), 671 (1999). 
${ }^{21}$ K. Terasaka, M. Y. Tanaka, S. Yoshimura, M. Aramaki, Y. Sakamoto, F. Kawazu, K. Furuta, N. Takatsuka, M. Masuda and R. Nakano, J. Plasma Phys. 81345810101 (2014)

${ }^{22}$ M. J. Goeckner, J. Goree and T. E. Sheridan, Rev. Sci. Instrum. 64(4) 996 (1993).

${ }^{23}$ V. Pigeon, N. Claire, C. Arnas and F. Doveil, Phys. Plasmas, 26(2), 023508 (2019)

${ }^{24} \mathrm{~S}$. Gerstenkorn, and P. Luc Atlas du spectre d'absorption de la molecule d'iode 14800-20000 cm-1. (Editions du Centre National de la Recherche Scientifique, Paris, 1978).

${ }^{25}$ E. Oyarzabal, A. B. Martin-Rojo and F. L. Tabarés, J. Nucl. Mat. 452(1-3), 37 (2014)

${ }^{26}$ L.A. Schwager and C.K. Birdsall, Phys. Fluids B: Plasma Phys. 2(5), 1057 (1990).

${ }^{27}$ J. P. Sheehan, The effects of Space-Charge Limited electron emission on the plasma sheath, University of Wisconsin-Madison (2012).
${ }^{28}$ T. Gyergyek and J. Kovačič and M. Čerček, Phys. Plasmas 17(8), 083504 (2010).

${ }^{29}$ M. D. Campanell and M. V. Umansky, Phys. Plasmas 24(5), 057101 (2017).

${ }^{30}$ R. Cimino, I. R. Collins, M. A. Furman, M. Pivi, F. Ruggiero, G. Rumolo and F. Zimmermann, Phys. Rev. Letters 93(1), 014801 (2004)

31 A. N. Andronov, A. S. Smirnov, I. D. Kaganovich, E. A. Startsev, Y. Raitses and V. I. Demidov, Joint INFN-CERN-EuCARDAccNet Workshop on Electron-Cloud Effects 2012 (2013).

${ }^{32}$ V. I. Demidov, S. F. Adams, I.D. Kaganovich, M. E. Koepke and I. P. Kurlyandskaya, Phys. Plasmas 22(10), 104501 (2015).

${ }^{33}$ F. X. Bronold and H. Feske, Phys. Rev. Letters 115(22), 225001 (2015).

${ }^{34}$ F. X. Bronold and H. Feske, Plasma Phys. Control. Fusion 59(1), 014011 (2016).

${ }^{35}$ X. Tian, R. K. Y. Fu, J. Chen, P. K. Chu and I. G. Brown, Nucl. Instrum. Methods Phys. Res. Sec. B: Beam Interactions with Materials and Atoms 187(4), 485 (2002). 


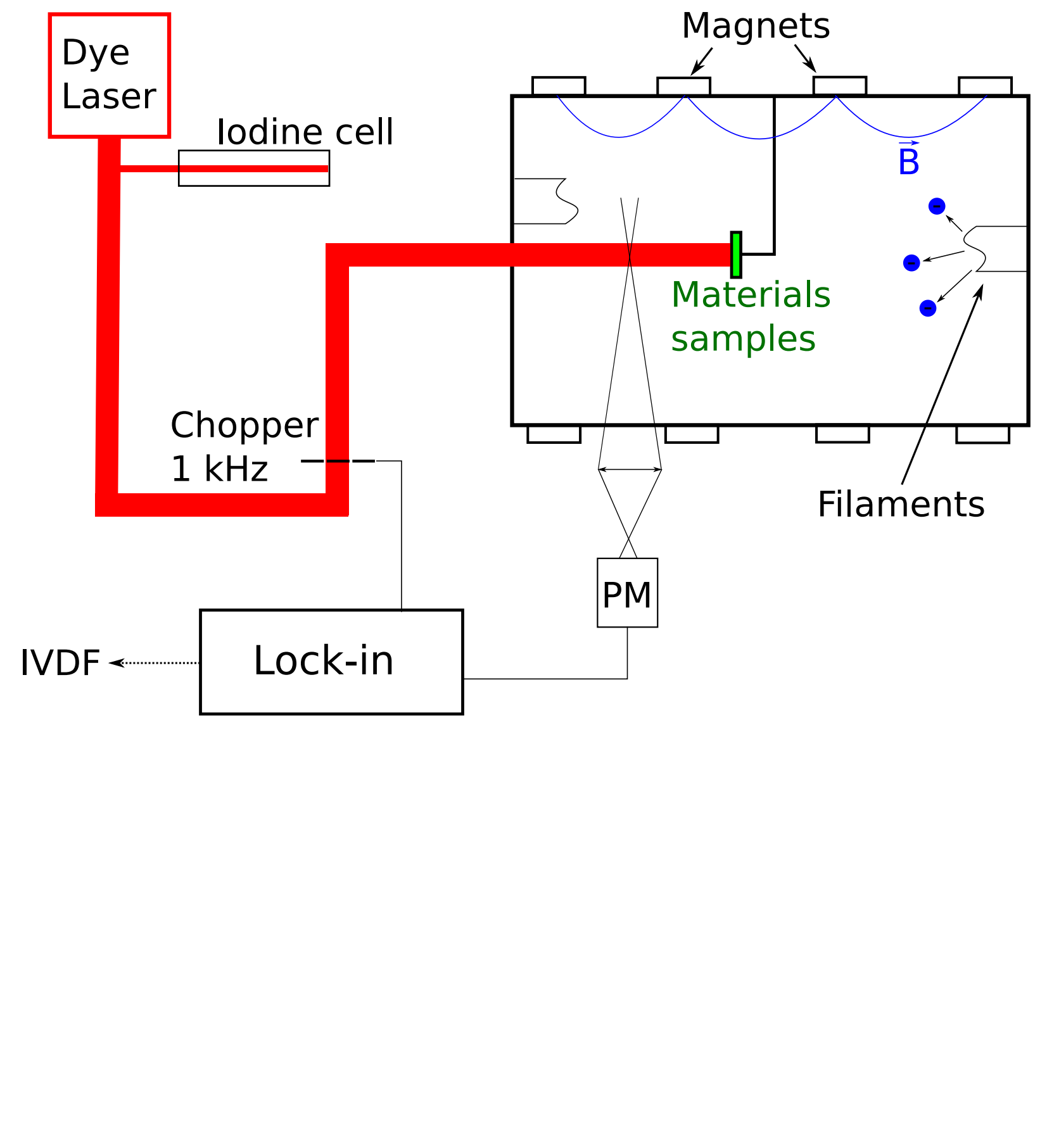




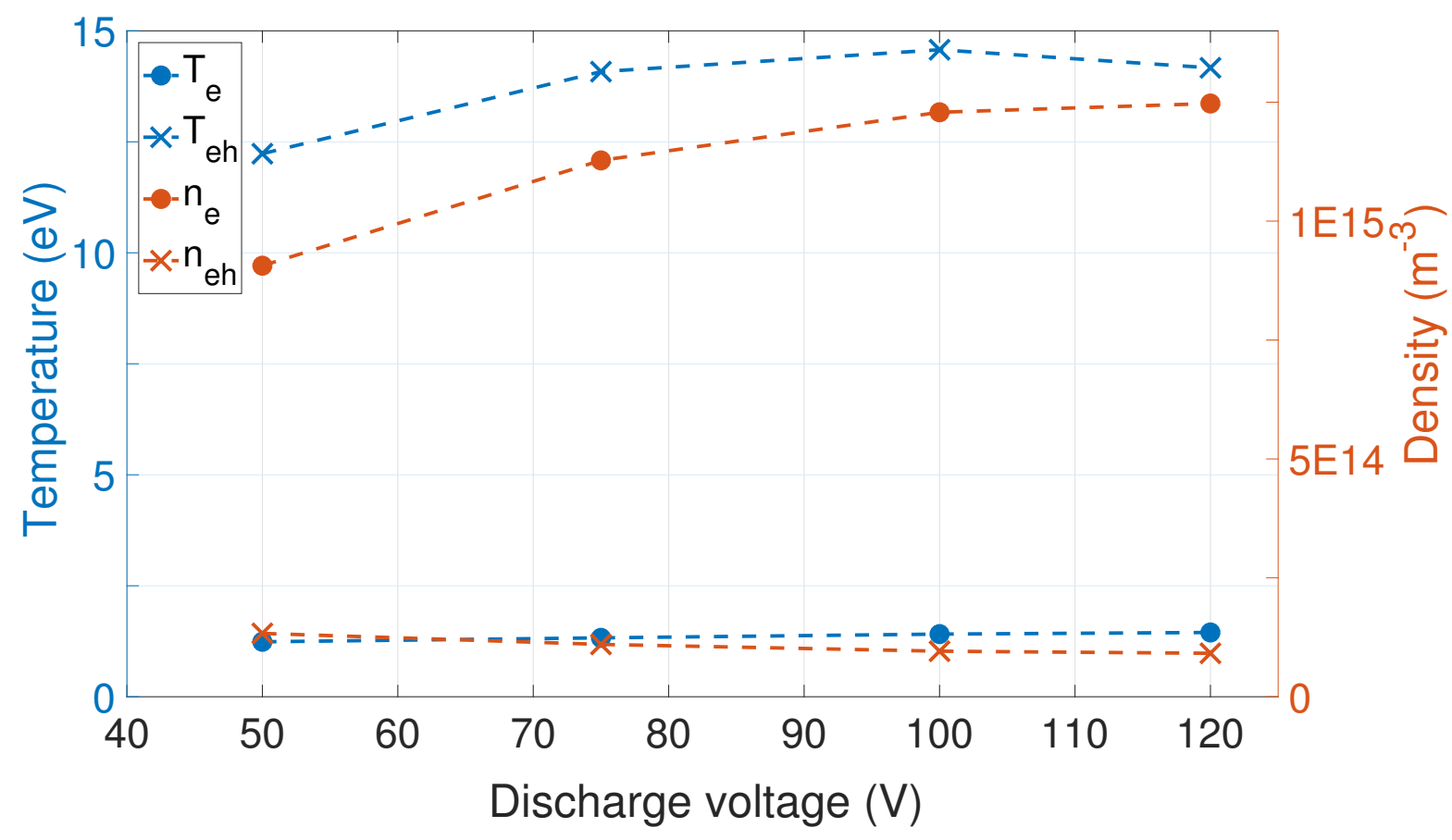




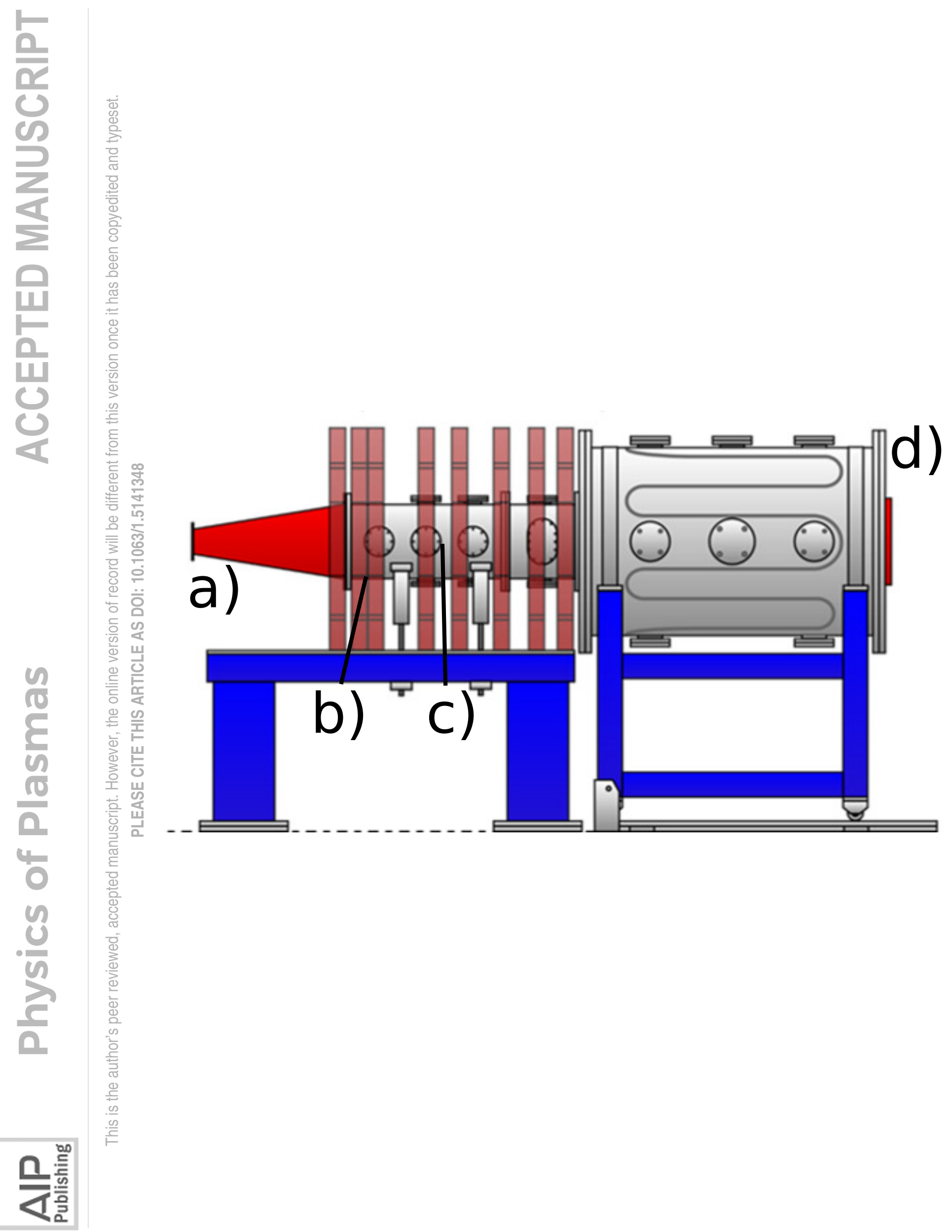



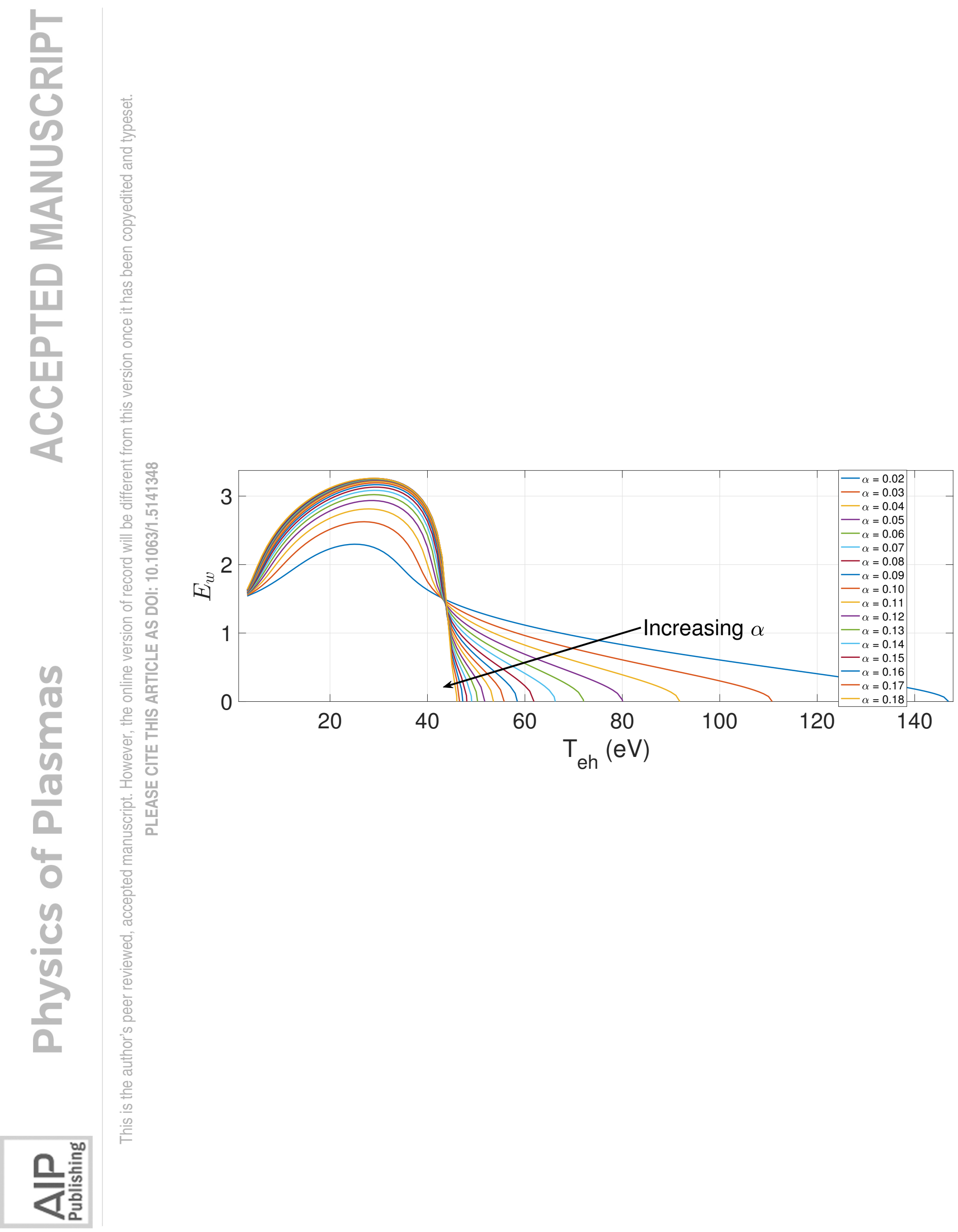


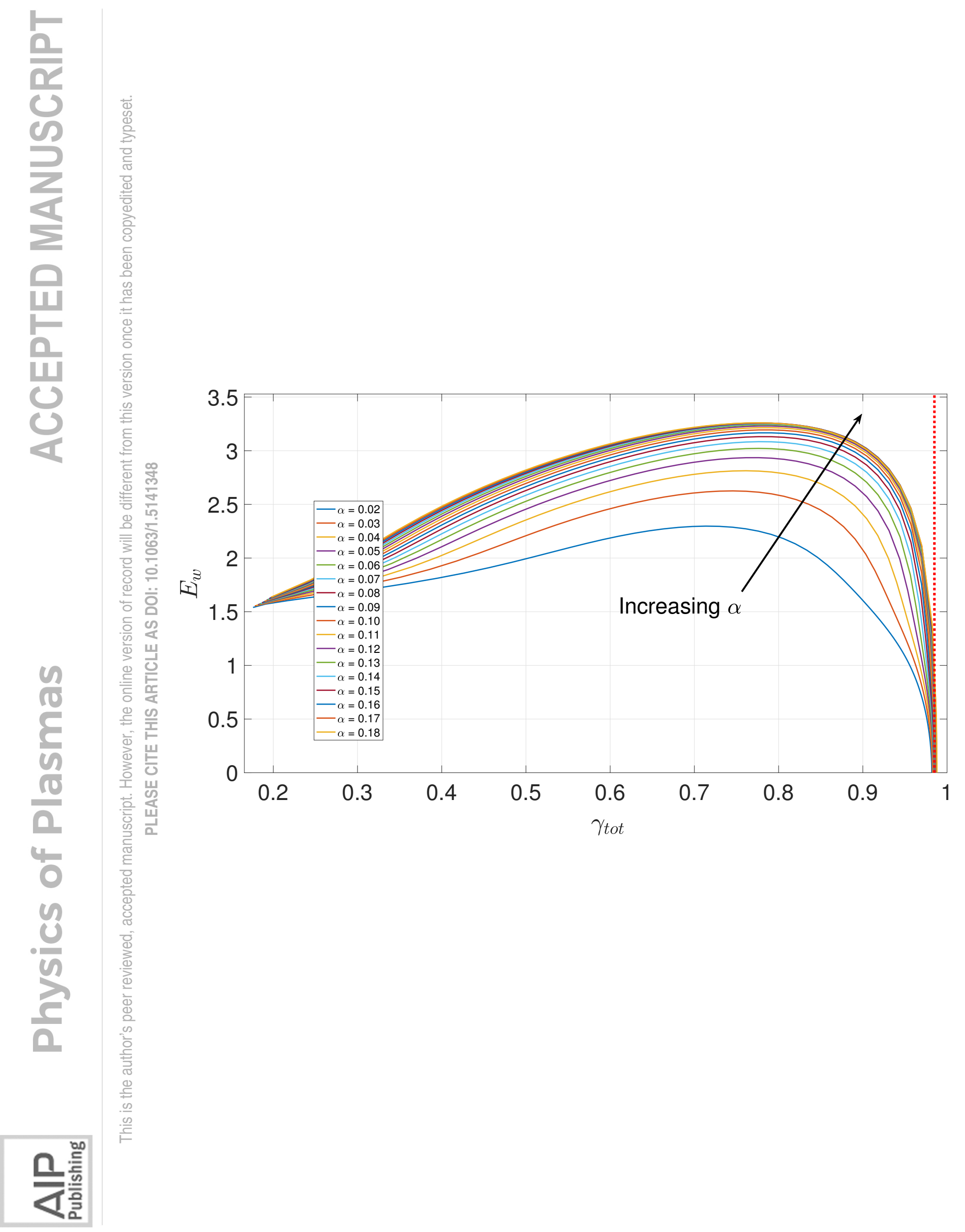




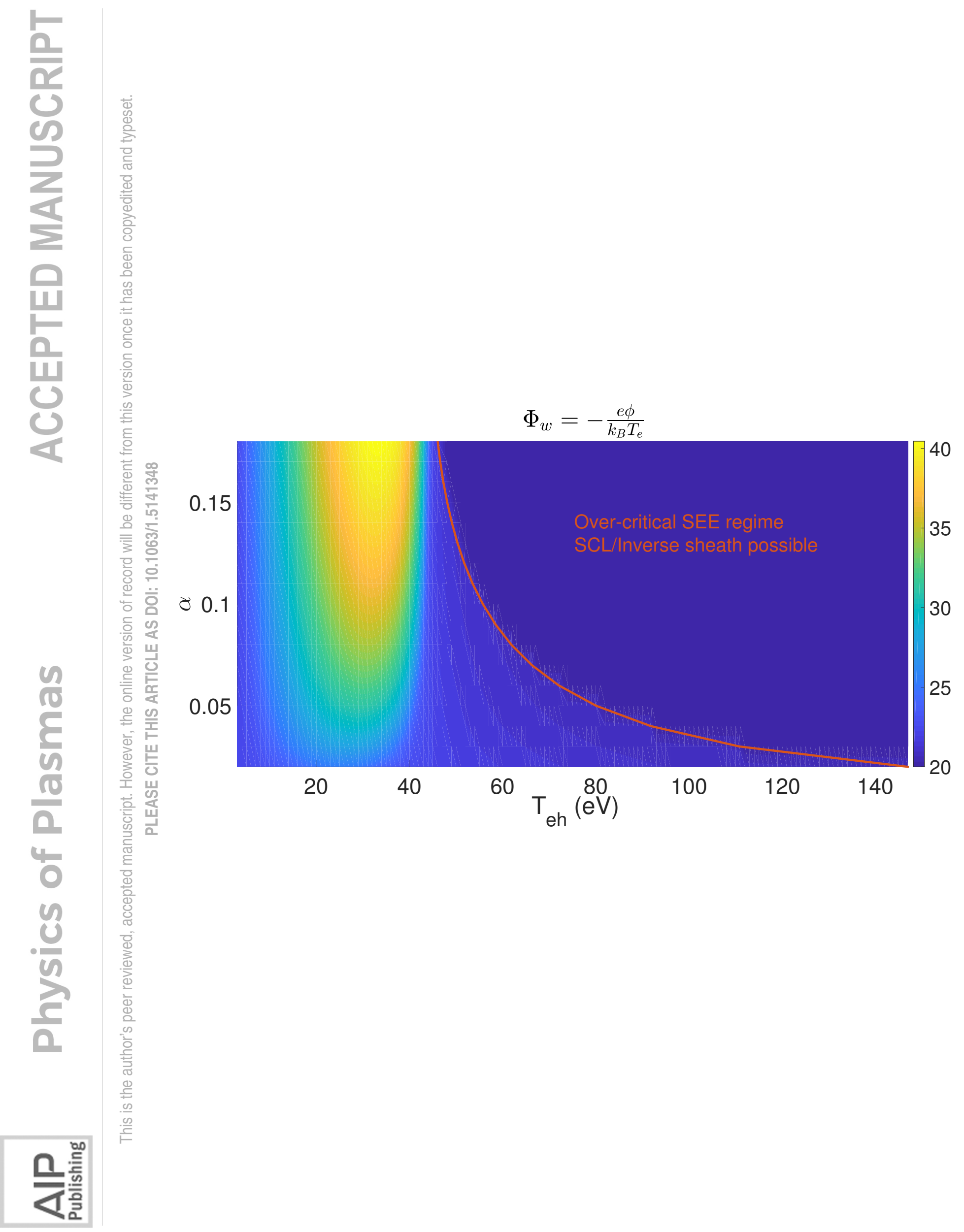




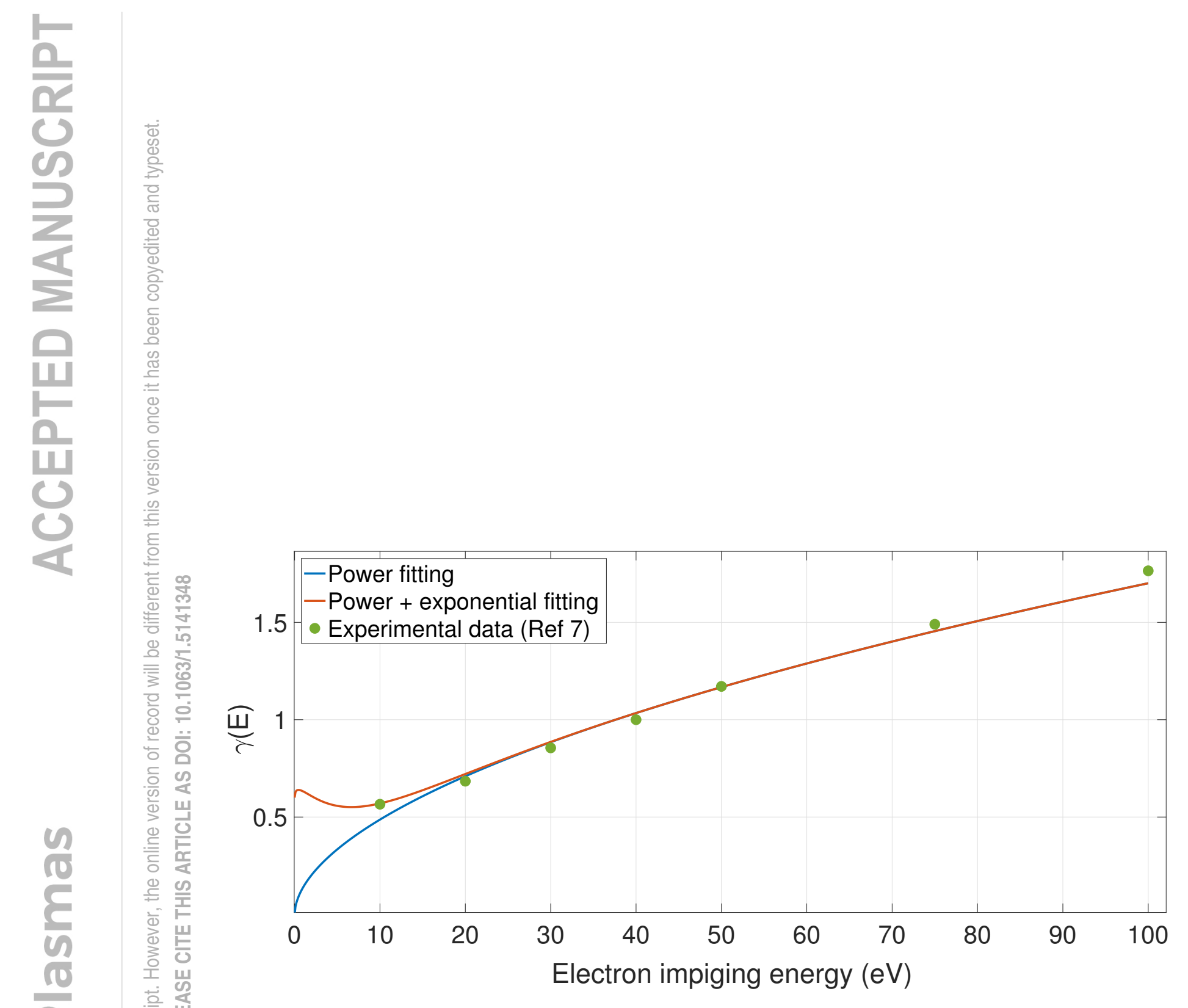




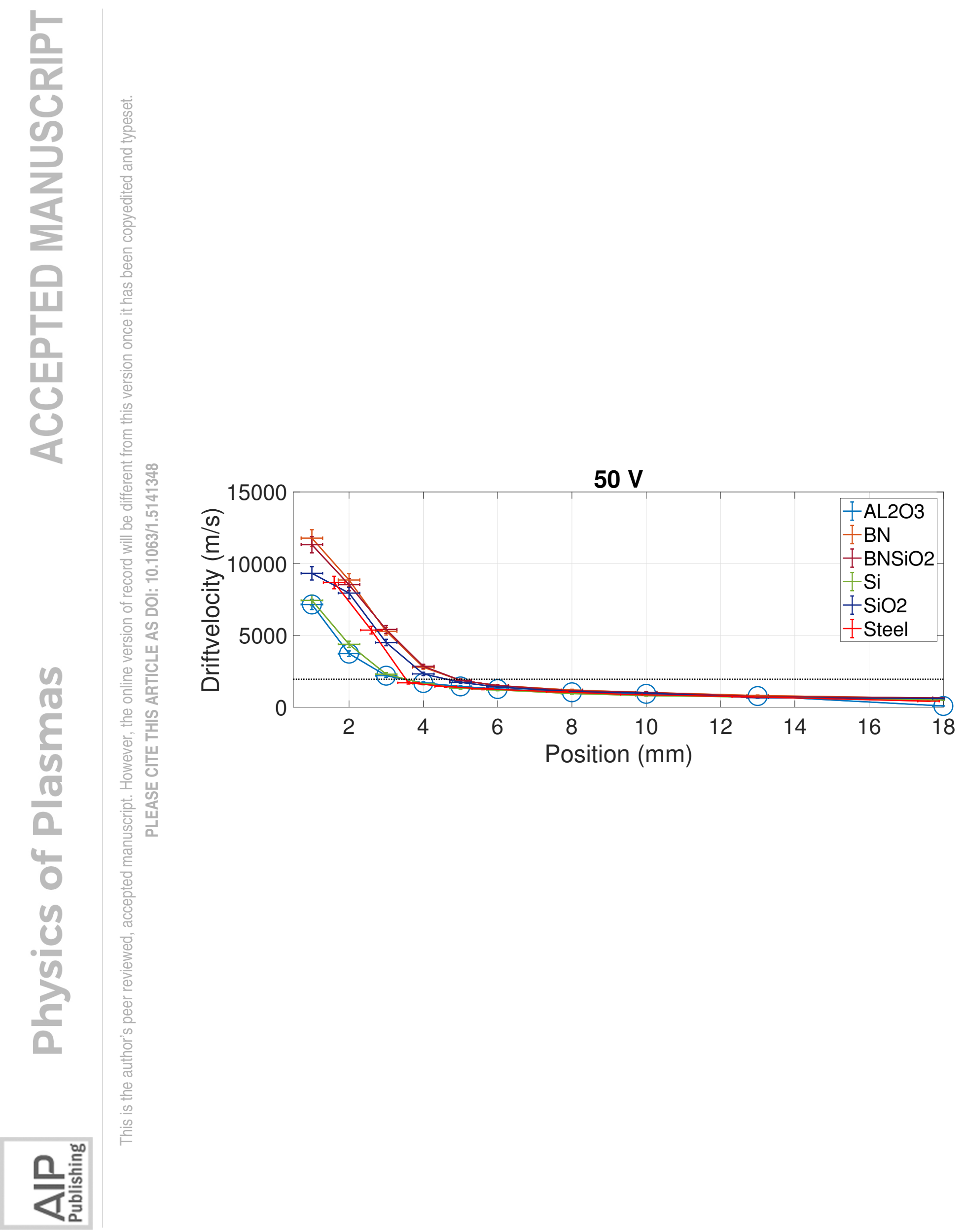


$75 \mathrm{~V}$

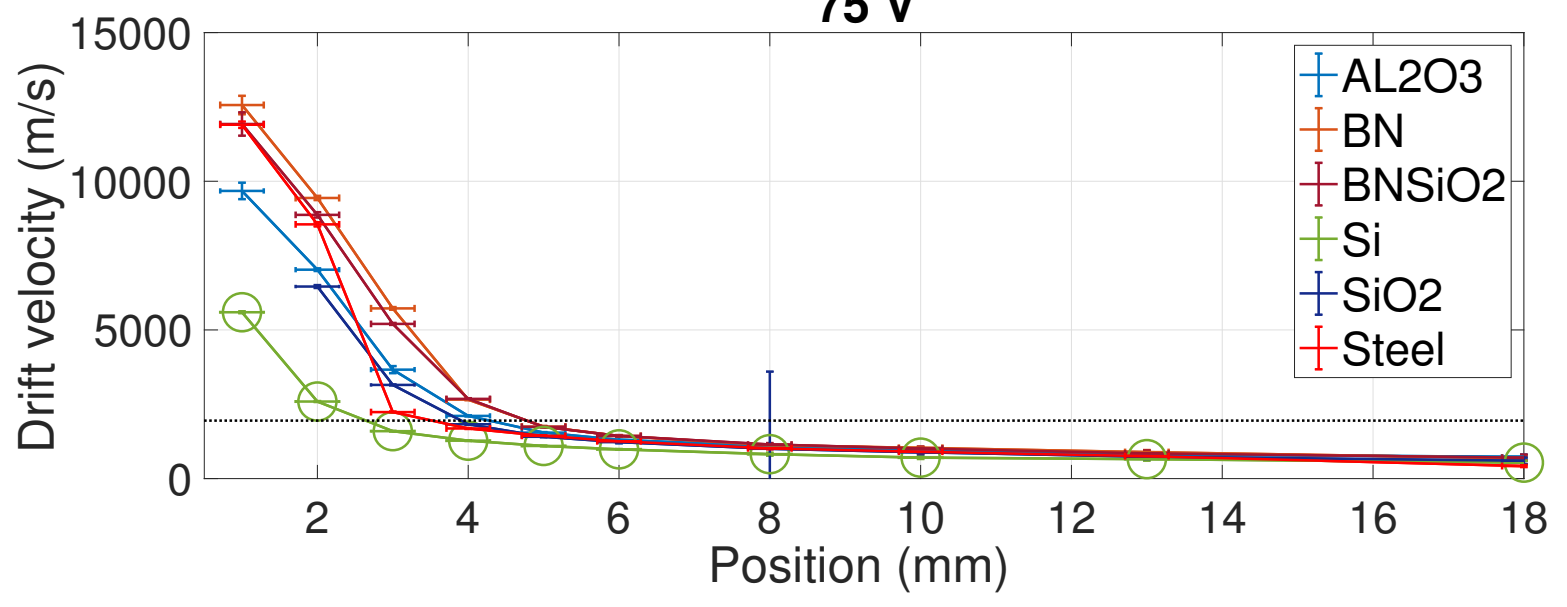




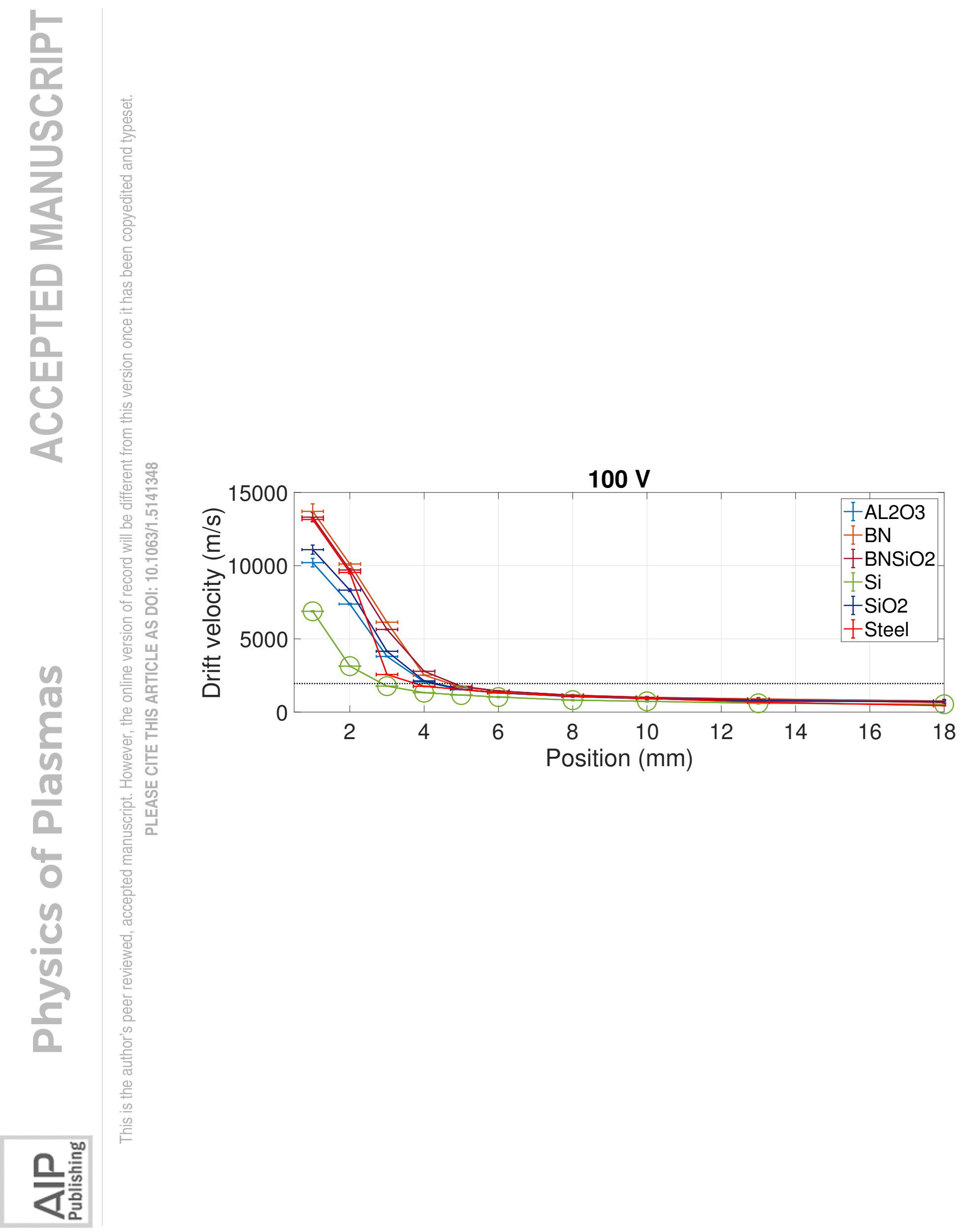




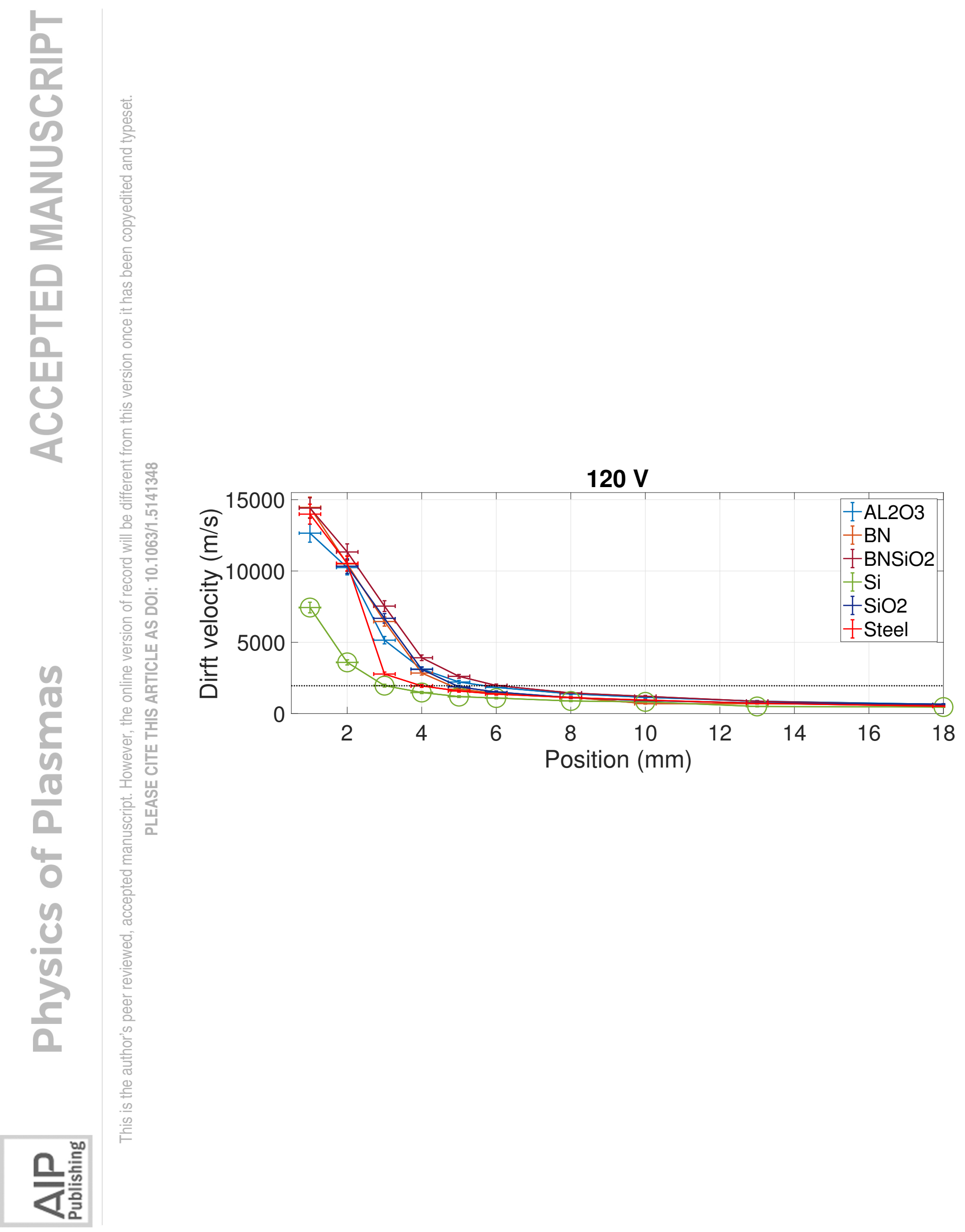




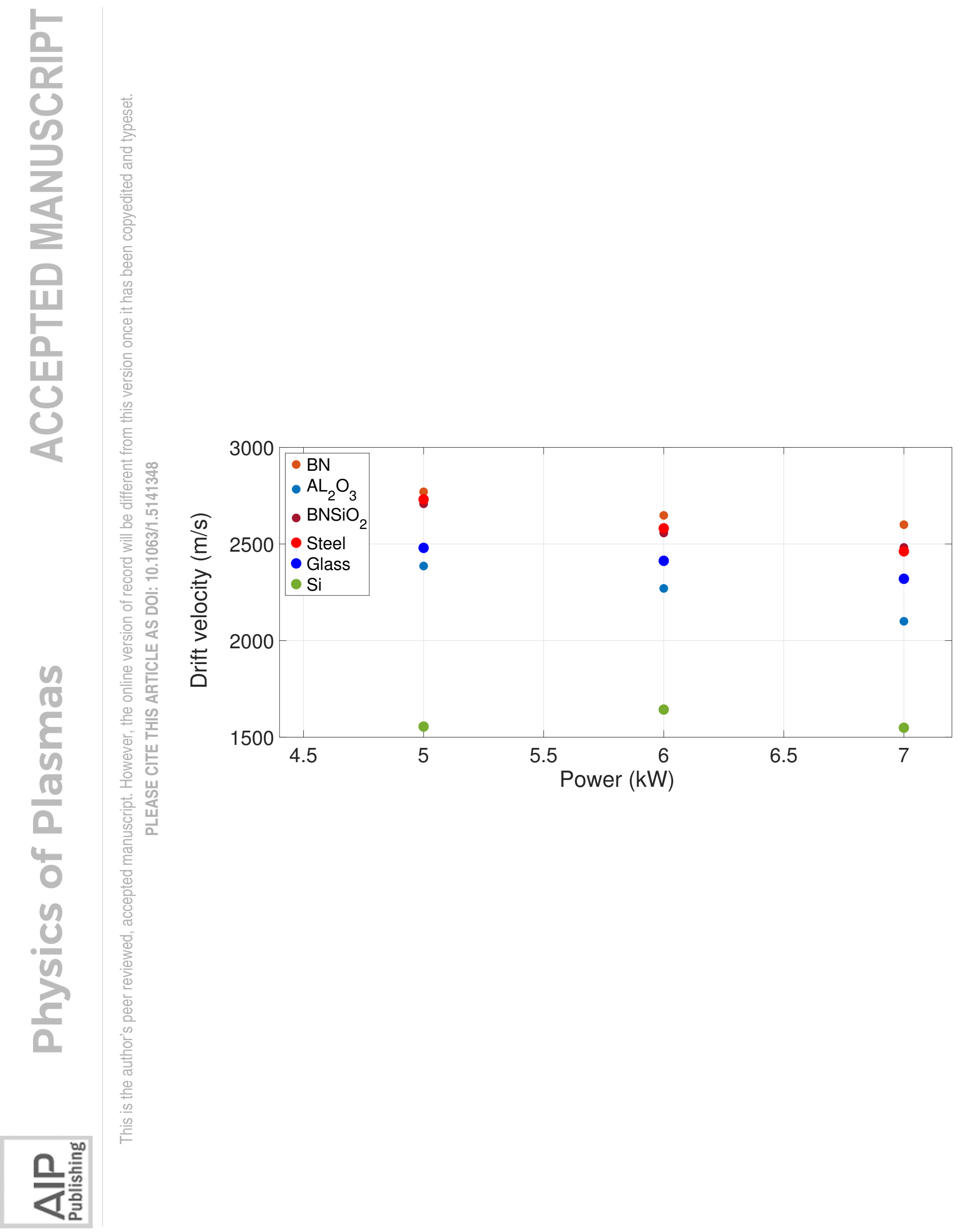




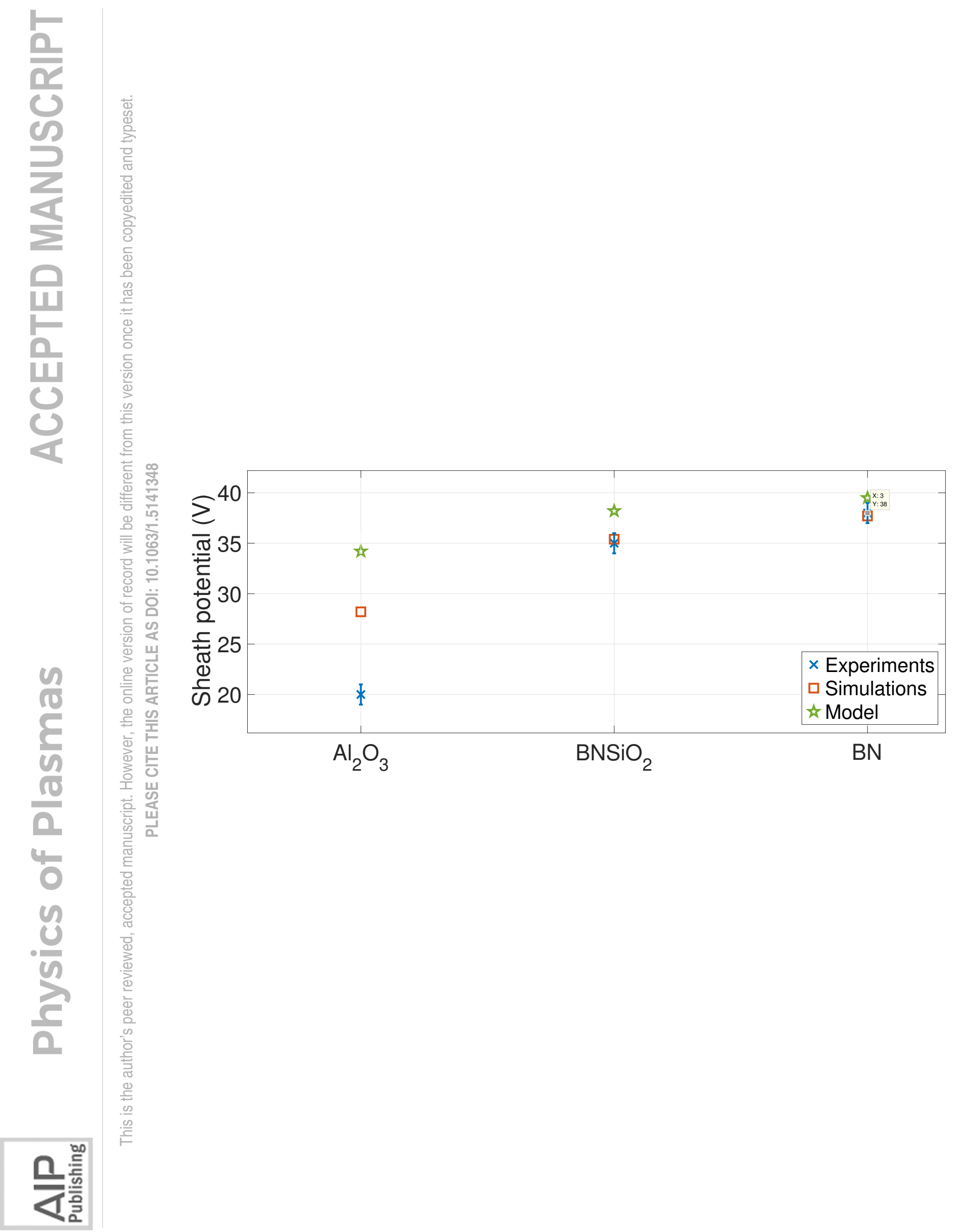

